\title{
Using fast homology search tools for protein sequence functional annotation: a comparison
}

Pronozin A.*, Genaev M., Afonnikov D.

Institute of Cytology and Genetics SB RAS, Novosibirsk, Russia

*e-mail: pronozinartem95@gmail.com

Key words: sequence functional annotation, GO terms, semantic similarity

Motivation and Aim: Annotation of the protein sequences by homology search and GO term transfer from highly homologous sequences is an important task for current genome and transcriptome sequencing projects. However, large size of sequence databases make homologous sequence search difficult in reasonable time. There exist tools that apply fast and ultrafast database search algorithms to find sequence homologs. We compare performance of the highly homologous sequence detection by several fast search tools applied for A.thaliana protein sequences represented in OrthoDB database with the sequence ranking obtained by ClustalW.

Methods and Algorithms: 27,636 sequences of the A. thaliana proteins from TAIR v10 [1]. We used orthologous genes from other organisms, as query homologs, which perform similar functions. The orthologous sequences identified from the OrthoDB database v10 [2]. We selected 9193 (contain 8,522,503 sequences) orthogroups containing A. thaliana proteins. Programs: BLASTP, BLASTP-fast, Diamond, Usearch ('ublast', 'usearch_local'), Mmseq2 were compared with sequence ranking obtained by ClustalW. For sequence list comparison, we used F1 and MAPK metric, compared the similarity of the GO terms associated with homologous hits by mens of semantic similarity (SS).

Results: The best values of performance metrics for single best hit observed for BLASTP (0.95). The performance decreased at $\mathrm{k}=5(0.75)$ and increased again to 0.80 at $\mathrm{k}=6-10$. These trends are similar for all tools. The result gene ontology (GO) comparison using F1 metric shows high similarity of the GO lists at $\mathrm{k}=1-10(0,97)$. Semantic similarity measure has almost no dependence on $\mathrm{k}$ for all gene ages. Computing time comparison demonstrated that Diamond have the smallest search time and the next is usearch_local method from Usearch.

Conclusion: To summarize, our results demonstrate that the optimal number of hits returned by fast search program for the functional annotation of the query sequence is 10 . The fast homology search tools are able to identify true best hits from large databases within $\mathrm{k}$ up to 20 with sufficient accuracy.

Acknowledgements: Supported by Russian Science Foundation grant 18-14-00293. The computational resources of the Joint HPC Facility 'Bioinformatics' was used with the support of the budget project No. 0324-2019-0040-C-01.

\section{References}

1. Mustafin Z. et al. Phylostratigraphic Analysis Shows the Earliest Origination of the Abiotic Stress Associated Genes in A. thaliana. Genes. 10.12, pp. 963, 2019.

2. Kriventseva E. et al. OrthoDB v10: sampling the diversity of animal, plant, fungal, protist, bacterial and viral genomes for evolutionary and functional annotations of orthologs. Nucleic Acids Research. 47.D1, pp. D807-D811, 2019. 